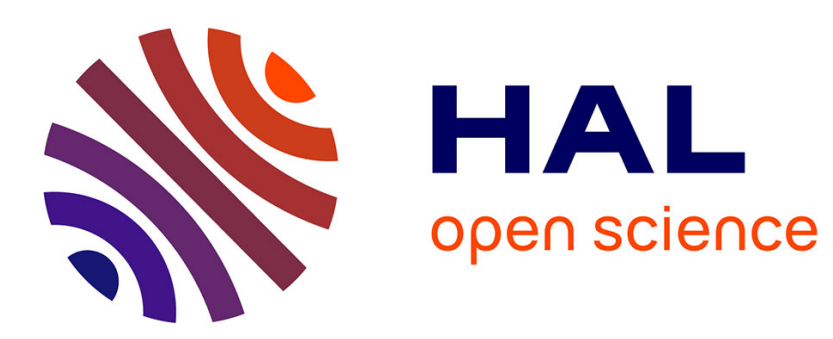

\title{
CRITICAL EXPONENTS IN NUCLEAR BREAKUP X. Campi
}

\section{To cite this version:}

X. Campi. CRITICAL EXPONENTS IN NUCLEAR BREAKUP. International Workshop on Semiclassical and Phase Space Approaches to the Dynamics of the Nucleus, 1987, Aussois, France. pp.C2151-C2-156, 10.1051/jphyscol:1987222 . jpa-00226488

\section{HAL Id: jpa-00226488 https://hal.science/jpa-00226488}

Submitted on 1 Jan 1987

HAL is a multi-disciplinary open access archive for the deposit and dissemination of scientific research documents, whether they are published or not. The documents may come from teaching and research institutions in France or abroad, or from public or private research centers.
L'archive ouverte pluridisciplinaire HAL, est destinée au dépôt et à la diffusion de documents scientifiques de niveau recherche, publiés ou non, émanant des établissements d'enseignement et de recherche français ou étrangers, des laboratoires publics ou privés. 


\title{
CRITICAL EXPONENTS IN NUCLEAR BREAKUP
}

\author{
X. CAMPI
}

Division de Physique Theorique, Institut de Physique Nucléaire, F-91406 Orsay Cedex. France

\section{Abstract}

The moments of the fragment size distribution are studied for single events in a nuclear fragmentation experiment. It is shown that this nuclear breakup has clean remanents of a critical phenomenon.

In recent years the study of cluster formation processes has become an important new field in statistical mechanics. This Eield concerns at present most branches of physics. We briefly discuss in this note how some of these new ideas and techniques can be applied to the nuclear fragmentation problem.

Many theoretical models of cluster formation processes have a critical behaviour, in the sense that for a fixed value of some variables (temperature, density, time...) typical quantities related to the cluster size distribution diverge (or become very large in finite systems). This behaviour appears in particular for some moments of the distribution. Consider an ensemble of $s_{0}$ elements and the distribution $n(s, \varepsilon)$ of clusters of size s at physical conditions defined by the variable $E$. We define the $k^{t}$ moment as

$$
M_{k}(\varepsilon)=\sum_{s} n(s, \varepsilon) s^{k}
$$

where the sum runs over all finite size clusters of an infinite system. In a finite system, we will take the prescription to sum over all present clusters, excluding the largest one. When the (infinite) system approaches the critical regime the singular part of the moments (1) behaves like

$$
M_{k}(\varepsilon) \sim|\varepsilon|^{\mu},(\varepsilon \rightarrow 0)
$$

where $\mu_{k}$ are the critical exponents [1-5]. The size of the largest cluster $s_{L}$ present in the system has also in some cases a critical behaviour

$$
s_{L} \sim|\varepsilon|^{\mu_{L}^{ \pm}},\left(\varepsilon \rightarrow 0^{ \pm}\right)
$$

defining a priori two new critical exponents $\mu_{L}^{ \pm}$. Some of these moments are related to important physical quantities and the corresponding critical exponents receive a specific notation. For 
example in ferromagnetic-paramagnetic transitions $M_{1} \sim\left|T-T_{c}\right|^{\beta}$ is related to the spontaneous magnetization, and $M_{2} \sim\left|T-T_{c}\right|^{-\gamma}$ to the magnetic susceptibility. In liquid-gas transitions, $M_{1}$ is related to the relative density difference between the liquid and its vapour and $M_{2}$ to the isothermal compressibility.

In formulas $(1-3)$ the variable $\varepsilon$ represents for example the distance to a critical temperature $\varepsilon=T_{c}-T$ in thermal phase transitions [1], to a critical"density" $\varepsilon^{c}=p-p_{c}$ in percolation theories [2], to a critical time $\varepsilon=t-t_{c}$ in dynamical aggregation theories [3]. The critical values $\left(T_{c}, p_{c}, t_{c}, \ldots\right)$ depend strongly on the nature of the physical substance or on the ingredients of the theoretical model (equation of state, lattice type..). In contrast, for a given order parameter and a fixed space dimension, the critical exponents are quite insensitive to the rature of the materials or to the details of the theoretical models. We can say that critical exponents are the fingerprints of the various types of critical phenomena, in the sense that when we know it for a given substance, we can decide to which class of phenomenon it belongs.

Many models predict near the critical regime a cluster size distribution of the type

$$
n(s, \varepsilon) \sim s^{-\tau} f\left(\varepsilon . s^{\sigma}\right)
$$

In general $f(0)=1$, i.e. at critical points the distribution is a pure power law. $\tau$ and $\sigma$ are two critical exponents.

We can now derive (2) for this particular distribution [2].

$$
\begin{aligned}
M_{k}(\varepsilon) & =\sum_{s=1}^{s_{\max }} n(s, \varepsilon) s^{k} \simeq \int_{0}^{s_{\max }} s^{k-\tau} f\left(\varepsilon, s^{\sigma}\right) d s \\
& =|\varepsilon|(\tau-k-1) / \sigma / \sigma \int_{0}^{\varepsilon \cdot s_{\max }^{\sigma}|z|^{(1+k-\tau) / \sigma} z^{-1} f(z) d z} \\
& =C_{k}^{ \pm}(\varepsilon) \cdot|\varepsilon|^{(\tau-k-1) / \sigma}
\end{aligned}
$$

where we have introduced the variable $z=\varepsilon . s^{*}$ and replaced the sum by an integral. We see that for an infinite system we recover (2) because the upper bound of the integral is infinity and the integral has a constant value $c_{k}^{t}$. In contrast, for a finite system the value of the integral depends on $\varepsilon$, and in particular goes to zero when $\varepsilon \rightarrow 0$. Than $M_{k}(\varepsilon)$ has always a finite value at critical point. As an example we show in figure 1 as a function of $\varepsilon$ the moment $k=3$ of a "typical" distribution $: n(s, \varepsilon)=$ $s^{2} \cdot{ }^{2} \exp \left(-11.75(z+0.2)^{2}\right), \quad z=\varepsilon \cdot s^{0.45}$ and $s_{\max }=100$. The full line represents $M_{3}(\varepsilon)$, the dashed-dot line the diverging term $|\varepsilon|^{{ }^{3}}$ and the dashed line the integral $c_{3}^{ \pm}(\varepsilon)$. We see in this example how the notion of critical behaviour remains valid in the vicinity of the critical point, but not too close to it.

A cluster size distribution of type (3) was first proposed by Fisher for his droplet model [3].

$$
\mathrm{n}(\mathrm{s}, \mathrm{T}) \sim \mathrm{s}^{-\tau} \cdot \exp \left(-\mathrm{const}\left|\mathrm{T}-\mathrm{T}_{\mathrm{c}}\right| \mathrm{s}^{\sigma}\right)
$$

with $\tau=7 / 3$ and $\sigma=2 / 3$ in 3 -dimensions. 
Percolation models [2] also follow (2) with great accuracy when $\varepsilon=p-p_{c} \rightarrow 0$. Here $p$ is the fraction of occupied sites or active bonds of a lattice, and $p_{c}$ the critical value at which the system "percolates" (i.e develops a single cluster that fills most of the lattice sites). In 3 -dimensions $\tau=2.2$ and $\sigma=0.45$, regardless of the percolation type (site or bond), lattice structure (simple cubic, body-centered cubic,..) or linkage prescriptions (nearest neighbours or longer range). Percolation can be seen as a static and purely geometrical modelization of very complex phenomena [5]. However the comparison of the physical reality with percolation is very tempty because this model is conceptually very simple and (numerical) calculations are easy, specially in small size systems. Also the predicted values for the exponents are close to those of Ising-magnet or lattice-gas models [5]. In addition percolation ideas have been recently introduced in nuclear fragmentation models [6]. We can say that percolation is the simplest cluster formation model showing a "reasonable" critical behaviour.

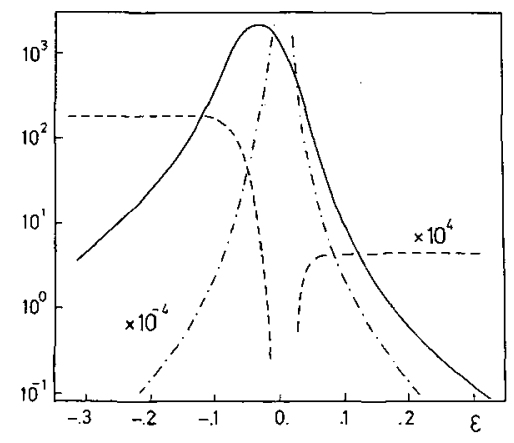

Fig. 1 - Plot of $M_{3}(\varepsilon)$ (full line) and its components in eq. (5):(dashed) $C^{ \pm}(\varepsilon)$ and $|\varepsilon|^{\mu_{3}}$ (dashed-dot) for a typical cluster size distribution.

In what follows we will compare experimental nuclear data on nucleus breakup to the predictions of an equivalent size percolation model, with the purpose to see that both systems show similar remanents of a critical behaviour, with similar critical exponents. We will take finite size percolation just as a reference model.

The piece of experimental data we analyze [7] consists in 370 collision events, in which $1 \mathrm{GeV}$. A Au projectiles break in lighter fragments when hit target nuclei in an emulsion. Event by event, the size (in fact the charge $1 \leqslant z \leqslant 79$ ) of all Au fragments has been measured [8]. Typical low energy fission events, as selected in ref.[8], are not included in the analysis. We discuss first how to show up the critical behaviour.

According to eqs. (2) and (3) in an infinite system we predict linear correlations in a log-log plot between the $M_{k}$ (and $s_{L}$ and $M_{k}$ ). In a small system we expect some distorsions due to the $\varepsilon$ dependence in $C_{\mu}(\varepsilon)$, eq. (5). We have studied the correlations $M_{3}-M_{2}, M_{3}-M_{2}$ and $s_{k}-M_{2}$ in ref. [7]. The correlations $M_{0}-M_{k}(k=1,2,3)$ are discussed in ref.[9]. Here we present the correlations $M_{s}-M_{2}$ and $s_{L}-M_{2}$. Because we ignore in the nuclear case what is $\varepsilon$ and we don't like to introduce any model dependence in our analysis, we work directly with single event distributions, i.e. for each collision event $j$ we calculate the moments 


$$
M_{k}^{j}=\sum_{s} s^{k} m^{j}(s)
$$

where $m^{1}(s)=0,1,2 \ldots$ is the number of fragments of size $s$ that appear in the event $j$. (The heaviest fragment $s_{L}$ is not included in the sum). We choose the normalization

$$
S_{k}^{j}=M_{k}^{j} / M_{1}^{j}
$$

Figure 2 shows the correlation $S_{5}^{J}$ against $S_{2}^{J}$. On the right (b), each point is a Monte Carlo simulation on a cubic lattice bond percolation model containing $6 \times 6 \times 6$ sites. The values of $p$ are chosen randomly distributed between 0 and 1 . Events close to critical region ( $p \simeq p_{c}$ ) are represented by points with the largest values of $s_{2}$ and $s_{9}$. Above and below the critical zone, points fall closer to origin. Nuclear fragmentation events are represented in fig. (2a). We see that for events with $s_{2} \lesssim 2$ the slope is about the same for the nuclear data as for the percolation simulation : $\lambda_{y / 2}=4.6 \pm .2$. This number is to be compared with $\lambda_{5 / 2}=4.75$ in infinite percolation models [2]. We can conclude within the present experimental uncertainties that the ratio $\mu_{5} / \mu_{2}$ is about the same in all cases. This is a rather unexpected result.

We also remark in figure(2a) a change in the slope for the events with $\mathrm{s}_{2} 22$. This was interpreted in ref.[7] as a manifestation of the finite size of the system affecting the most critical events as discussed with formula (5). A closer investigation reveals a different origin. Most of the events that populate this region have two very big fragments, plus many $s=1$ or $\mathbf{s = 2}$ fragments. Obviously this events do not fit with a size distribution of type (4), what explains the anomalous slope. In contrast, these events seem to correspond to the prediction of D.H.E. Gross $[10,11]$ of a medium excitation energy "fission like" fragmentation.

The correlation $s_{L}-s_{3}$ is plotted in figure 3. Here each point represents the average over two or more events with the $s_{a m e} s_{L}$. Again we observe similar correlations, with similar slopes. The upper branch of the curves concern events with $p>p_{c}$ (b) or "gentle" nuclear collision events (a). The lower branch represents events below $p_{c}$ (b) or violent collisions (a). The slope of this branch is $\lambda_{1 / 2} \approx 1.2 \pm 0.2$ in both cases, in agreement with the predicted value in infinite percolation $\lambda_{L / 2}=1+\beta / \gamma=1.26$. About the same value is predicted for (infinite) thermal phase transitions, but $\lambda_{L / 2}=1.5$ in a mean field approximation.

We summarize. The validity of the "universal" scaling relation (4) has been tested for nuclear fragment size distributions. A finite size percolation model has been used as simple test model. Using moments technique we have seen in both cases similar remanents of the behaviour predicted by this equation, with similar ratios of critical exponents. These ratios are close to those of percolation and those of Ising-magnet or lattice-gas models in infinite systems, but different from the mean field approximation. In any case the ratios of critical exponents we have deduced from experimental data put severe new constraints on static or dynamic nuclear fragmentation models. 

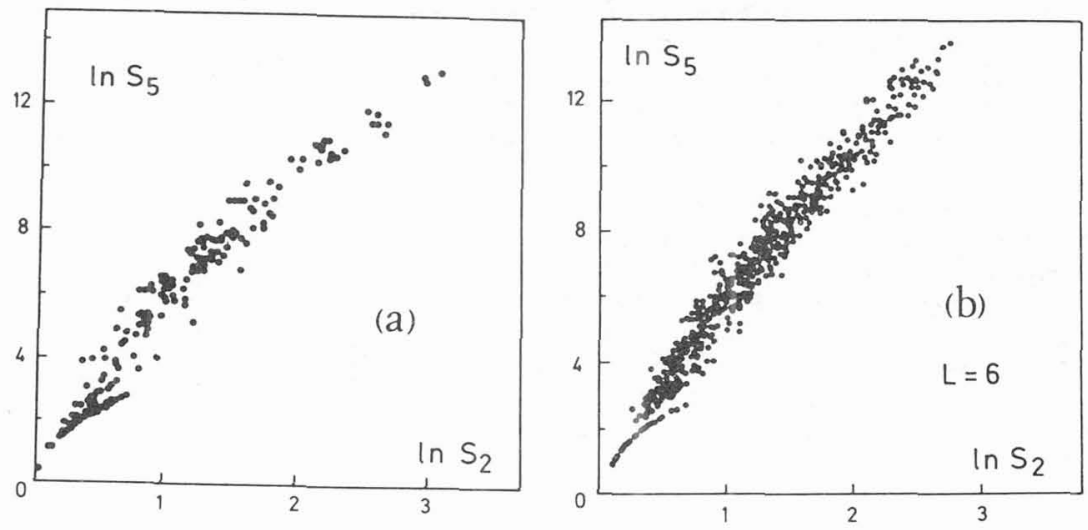

Fig.2 - Single event moments $S_{5}^{j}$ plotted against $s_{2}^{j}$, for nucleus break-up (a) and for a Monte Carlo simulation in a cubic bond percolation model containing 216 sites and randomly distributed values of $0<p<1$ (b).
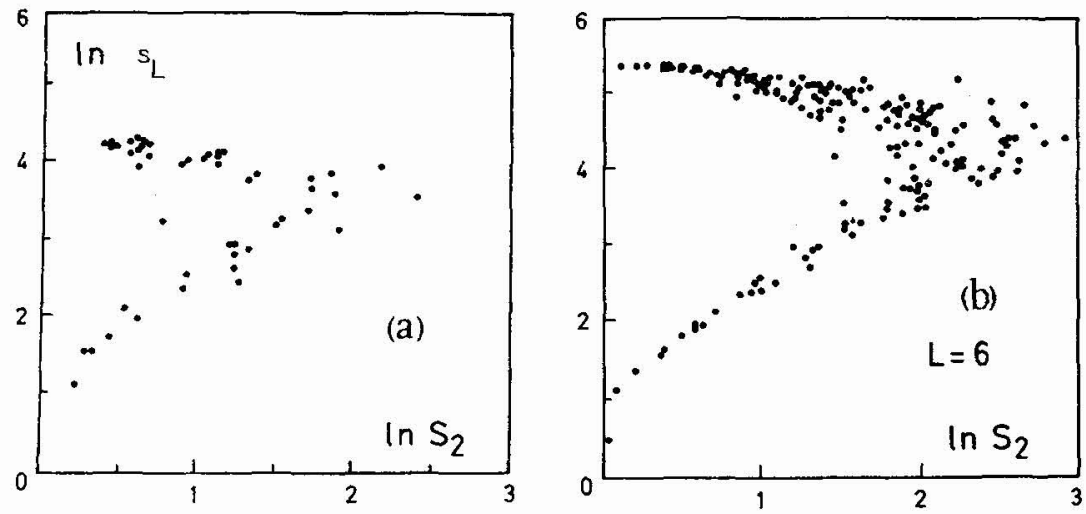

Fig.3 - The size of the largest cluster produced per event, as a function of $s_{2}$. Each points represents the average over events with same $s_{L}$. a) Largest nuclear fragment charge. Average over 376 events. b) Largest cluster size in a cubic bond percolation model containing 216 sites and for randomiy distribution values of $0<p<1$. Average over 4000 events. Only the slopes of the curves can be compared because of different system size.

\section{References}

[1] C. Domb and M.S. Green in Phase Transitions and Critical Phenomena, vol. $1-6$, (New York Academic 1972-1976).

[2] D. Stauffer, Introduction to percolation theory, Taylor and Francis, London 1985.

[3] H.J. Herrmann, Phys. Rep. 136 (1986) 153.

[4] M.E. Fisher, Physics (N.Y.) 3 (1967) 255.

[5] H.E. Stanley in Introduction to Phase Transitions and Critical Phenomena (OUP, Oxford 1971). 
[6] X. Campi and J. Desbois, GSI Report 85-10, 1985; Contribution to the XXIII Bormio Meeting 1985.

W. Bauer, D.R. Dean, U. Mosel and U. Post, Phys. Lett. 150B (1985) 53 ; Nucl. Phys. A452 (1986) 699.

T.S. Biro, J. Knoll and J. Richert, Nucl. Phys. A459 (1986) 692 .

J. Nemeth et al., Z.Phys. A325 (1986) 347.

J. Desbois, Nucl. Phys. in press.

[7] X. Campi, J. Phys. : Math. Gen.19 (1986) L917.

[8] C.J. Waddington and P.S. Freier, Phys. Rev. C31 (1985) 888.

[9] X. Campi, Contribution to the XXV Bormio Meeting 1987.

[10] D.H.E. Gross, Contribution to this Conference.

[11] X.Z. Zhang, D.H.E. Gross, S.Y. Xu and M. Zheng, Nucl. Phys. A461 (1987) 641, and ref, therein. 